\title{
Auswirkungen des Staatenwettbewerbs auf die Steuergerechtigkeit
}

Eines der wichtigsten Fundamente der Gesellschaft und jenseits der Nationalstaaten ist das Recht. Es ist das verbindende Band der Länder und Kulturen und das Ergebnis einer langen Überlieferung. ${ }^{1211}$ Dieses verbindende Band wird zerschnitten, wenn durch den Staatenwettbewerb unterschiedliche Steuerbelastungen entstehen.

Mit der Festschreibung des Gleichheitsgebotes in der Weimarer Reichsverfassung vom 11. August 1919 wurde ein Jahrhunderte währender Streit beendet. Die letzten absolutistischen Privilegien, als auch alle Arten von unbegründeten steuerlichen Befreiungen und Vergünstigungen wurden für unzulässig erklärt und aufgehoben. Die internationalen Verflechtungen in der zweiten Hälfte des 20. Jahrhunderts jedoch - insbesondere in wirtschaftlicher Hinsicht - hatten nicht nur Auswirkungen auf die Finanzierungsgrundlagen der Nationalstaaten, sondern schufen auch mannigfaltige Möglichkeiten, die steuerlichen Belastungen zu mindern und auch völlig zum Wegfall zu bringen. Damit wurden nicht nur die Grundsätze der Allgemeinheit und Gleichmäßigkeit verletzt, sondern insbesondere der Besteuerung nach der Leistungsfähigkeit der Boden entzogen.

Auf der Ebene des Staatenwettbewerbs entstehen Gerechtigkeitsprobleme, wenn Steuerpflichtige durch Verlagerung eines mobilen Besteuerungsgutes (z. B. Kapital) in Niedrigsteuerländer weniger Steuern zahlen als Steuerpflichtige mit immobilen Besteuerungsgütern (z. B. Arbeit). Die unterschiedlichen Mobilitätsbedingungen

1211 Stolleis, Michael: Das verbindende Band, in: Rotary Magazin für Deutschland und Österreich, Juni 2017, S. 40 f. 
bewirken, dass der Standortwettbewerb nicht von allen Produktionsfaktoren gleichermaßen in Anspruch genommen werden kann; der Boden kann ihn nicht wahrnehmen, die Arbeit nur in geringem Umfang. Sach- und insbesondere Geldkapital als Hauptnachfragende des Standortwettbewerbs nehmen zwar die gleichen staatlichen Gesamtleistungen in Anspruch, können jedoch ganz oder teilweise der Besteuerung entzogen werden. Dies ist mithilfe von Staaten möglich, die sich zu ihrem eigenen Vorteil unkooperativ gegenüber anderen Staaten verhalten. Dies ist auf dreierlei Weise denkbar: Erstens durch staatliche Privilegierungen, zweitens durch internationale Steuergestaltung und drittens durch internationale Steuerhinterziehung. ${ }^{1212}$

\subsection{Privilegierte Besteuerung für mobile Einkünfte}

Bereits gegen Ende des 19. Jahrhunderts entwickelte sich steuerinduzierte Migration zu einer glaubhaften Drohung. Die Mobilitätsschranken fielen, als mobiles Kapital verstärkt der Besteuerung unterzogen wurde und lokale Gerichtsbarkeiten weitgehende fiskalische Autonomie erhielten. Offenbar überstieg die stark ansteigende steuerliche Belastung eine Schwelle, ab der steuerliche Aspekte ausschlaggebend für Wohn- und Standortentscheidungen wurden. Kommunen schienen um die Jahrhundertwende bemüht zu sein, wohlhabende Steuerzahler durch eine gezielte Kombination von Steuer- und Ausgabenpolitik anzuziehen. ${ }^{1213}$

Da mobile Produktionsfaktoren - insbesondere Geldkapital - relativ leicht und schnell verlagert werden können, besteht für Staaten der Anreiz, dies gegenüber immobilen Produktionsfaktoren zu privilegieren. Dies geschieht dadurch, dass Kapitaleinkünften ein niedrigerer Preis für die staatliche Gesamtgegenleistung abverlangt wird als den auf immobilen Faktoren beruhenden Einkünften. ${ }^{1214}$

Die Einführung einer 25 prozentigen Abgeltungsteuer auf Kapitaleinkünfte ${ }^{1215}$ ab dem Jahr 2009 sollte die Attraktivität und Wettbewerbsfähigkeit des Finanzplatzes Deutschland verbessern und einen Anreiz schaffen, um abgeflossenes Kapital

1212 Valta, Matthias: Das Internationale Steuerrecht zwischen Effizienz, Gerechtigkeit und Entwicklungshilfe, Tübingen 2014, S. 171-174.

1213 Vgl. Spoerer, Mark: Steuerlast, S. 176-192.

1214 Ebd., S. $174 \mathrm{f}$.

1215 Bereits im Jahr 1952 wurde bei den Einkünften aus Kapitalvermögen die progressive Einkommensteuer durch eine Kapitalertragsteuer von 30 Prozent ersetzt. Vgl. das Gesetz zur Förderung des Kapitalmarkts vom 15. Dezember 1952, BGBl. I 1952, S. 793. 
wieder zurück ins Land zu holen. Diese Maßnahme wird seit der Einführung als ungerecht kritisiert, da der progressive Einkommensteuersatz mit bis zu 45 Prozent Grenzsteuerbelastung bei den übrigen Einkunftsarten beibehalten wurde. $\mathrm{Zu}$ bedenken ist jedoch, dass - im Gegensatz zur Privilegierung von Zinsen - der effektive Steuersatz bei ausgeschütteten Gewinnen von Körperschaften unter Einbeziehung der Vorbelastung durch Gewerbe- und Körperschaftsteuer rund 48 Prozent beträgt. ${ }^{1216}$ Dividenden werden damit keineswegs privilegiert, sondern stärker als z. B. Arbeitseinkommen und Zinsen belastet.

Bei einer Abkehr von der Abgeltungsteuer wären alle Steuerzahler, die den Sparer-Pauschbetrag für ihre Zinseinkünfte bereits ausgeschöpft haben und deren Einkommensteuergrenzsatz über 25 Prozent liegt, direkt betroffen. Bei Einzelveranlagung ist das bereits bei einem zu versteuernden Einkommen von 16.000 Euro der Fall, da der Einkommensteuergrenzsatz 25,12 Prozent beträgt, sodass auch kleine und mittlere Einkommen von Mehrbelastungen betroffen wären. ${ }^{1217}$

Fernerhin ist auch anzumerken, dass mit der Einführung der Abgeltungsteuer die Bemessungsgrundlage verbreitert wurde ${ }^{1218}$ weil die tatsächlichen Werbungskosten nicht mehr abgezogen werden können und Veräußerungsgewinne unabhängig von der Haltedauer von Wertpapieren seit 2009 besteuert werden.

Auch die Besteuerung von Kapitalgesellschaften wurde im Jahr 2008 von einer Durchschnittsbelastung von ca. 38 Prozent auf nominell ca. 29 Prozent abgesenkt. ${ }^{1219}$ Eine weitere Privilegierung fand mit der Tonnagebesteuerung der internationalen Seeschifffahrt statt. Der Bundesfinanzhof hat den Privilegierungscharakter - mit einer Steuerbelastung der effektiven Gewinne von ca. 5 Prozent - ausdrücklich benannt. ${ }^{1220}$

Die hier aufgeführten Beispiele einer privilegierten Besteuerung verstoßen gegen den Gleichheitsgrundsatz, werden jedoch im internationalen Steuerwettbewerb als

1216 Deutsches Steuerzahlerinstitut des Bundes der Steuerzahler: Bausteine für eine Reform des Steuersystems. Das DSI-Handbuch Steuern, Berlin 2013, S. 112.

1217 Bei einem zu versteuernden Einkommen von z. B. 20.000 Euro steigt der Einkommensteuergrenzsatz auf 26,95 Prozent.

1218 Auch durch die Einschränkung der Verlustverrechnung ist die Bemessungsgrundlage erweitert worden, was sich nach Hey verfassungsrechtlich nicht rechtfertigen lässt. Vgl. Hey, Johanna: $\$ 8$ Einkommensteuer, in: Tipke, Klaus/Lang, Joachim (Hrsg.): $\$ 8$, Anm. 500.

1219 Vgl. Kavic, Claudia/Vogel, Andrea: Der tatsächliche Verlauf des Unternehmensteuertarifs nach der Unternehmensteuerreform 2008, in: Deutsches Steuerrecht, 12 (2008), S. 573.

1220 BFH vom 20. 11. 2006, VIII R 33/05, BStBl II 2007, S. 261, 263. 
notwendig und legitim angesehen. ${ }^{1221}$ Dennoch bleibt die Frage der Gerechtigkeit bestehen, denn von gleichmäßiger Steuerlastverteilung, die auf dem Leistungsfähigkeitsprinzip beruht, kann nicht gesprochen werden.

\subsection{Internationale Steuergestaltung}

International operierenden Unternehmen stehen eine Reihe von Steuergestaltungsmöglichkeiten zur Verfügung, die darauf hinauslaufen, möglichst hohe Gewinne in Niedrigsteuerländern anfallen zu lassen, steuermindernde Verluste hingegen in Hochsteuerländern. Einige von diesen Methoden sind illegal, aber die meisten sind vollkommen gesetzeskonform. ${ }^{1222}$

\subsubsection{Die Unterkapitalisierungsmethode}

Bei der Unterkapitalisierungsmethode (thin capitalisation), auch Fremdfinanzierungsmethode genannt, wird eine international operierende Firma in einem Land - z. B. in Deutschland - mit vergleichsweise hohen Steuersätzen mit geringem Eigenkapital ausgestattet - also unterkapitalisiert - und finanziert sich im Wesentlichen mit Fremdkapital bei einer anderen konzerneigenen Gesellschaft. Die Schuldzinsen mindern den Gewinn der deutschen Firma, die Zinserträge unterliegen dem niedrigeren Steuersatz des Landes, in dem die Konzerngesellschaft ihren Sitz hat. ${ }^{1223}$ Diese Form der Gewinnverschiebung wurde durch die Steuerreform 2008 jedoch eingeschränkt, da seitdem die Zinsen in Deutschland nicht mehr voll abgesetzt werden können.

1221 Valta, Matthias: Das Internationale Steuerrecht, S. 176. Das Bundesfinanzministerium weist darauf hin, dass die Stellung Deutschlands im internationalen Vergleich seit der Absenkung des deutschen Körperschaftsteuersatzes in 2008 auf 15\% deutlich wettbewerbsfähiger geworden ist. Bundesministerium der Finanzen: Die wichtigsten Steuern im internationalen Vergleich 2015-Ausgabe 2016. Übersicht 3: Körperschaftsteuersysteme und Anteilseignerbesteuerung 2015, auf: http://www.bundesfinanzministerium.de/Content/DE/Downloads/Broschueren_Bestellservice/2016-05-13-wichtigsten-steuern-im-internationalen-vergleich-2015.pdf;jsessionid=448241F9200587 145746 DEE9F80D439F?__blob=publicationFile\&v=8, (letzter Zugriff: 02.03.2017).

1222 Liebert, Nicola: Steuergerechtigkeit in der Globalisierung. Wie die steuerpolitische Umverteilung von unten nach oben gestoppt werden kann, Münster 2011, S. $143 \mathrm{f}$.

1223 Ebd., S. 145. 


\subsubsection{Gewinnverlagerungen mithilfe der Nutzung von Patenten, Marken oder Urheberrechten}

Analog zur Unterkapitalisierungsmethode können die Kosten für die Nutzung von immateriellen Wirtschaftsgütern im Hochsteuerland abgezogen und in Niedrigsteuerländern als Ertrag bei verbundenen Unternehmen zu geringeren Sätzen versteuert werden. Als Beispiel für eine derartige Steuergestaltung kann die Firma Amazon angeführt werden, die Verträge mit Drittanbietern, die Amazon zur Abwicklung ihrer Geschäfte nutzten, nicht mehr in den USA, sondern über Luxemburg abwickelt. Dort werden zwar die Unternehmensgewinne mit 29 Prozent belastet, Einkünfte, die mit geistigem Eigentum erwirtschaftet werden, werden jedoch unter bestimmten Umständen bis zu 80 Prozent von der Bemessung des zu versteuernden Gewinns ausgenommen. Nach Recherchen der Nachrichtenagentur Thomson Reuters soll es Amazon mithilfe dieser Maßnahmen gelungen sein, rund zwei Milliarden Dollar einzusparen, um diese für die Expansion des Unternehmens zu nutzen. ${ }^{1224}$

\subsubsection{Manipulationen durch interne Verrechnungspreise}

Die Transferpreismethoden basieren auf der Manipulation der internen Verrechnungspreise zwischen Konzerngesellschaften in verschiedenen Ländern. Gewinnverlagerungen sind dergestalt möglich, dass das Unternehmen Produkte und Dienstleistungen an das in einem Niedrigsteuerland sitzende verbundene Unternehmen unter den marktüblichen Preisen anbietet, oder umgekehrt seine Vorprodukte zu überhöhten Preisen von dort bezieht. Nach einer Schätzung der Organisation für wirtschaftliche Zusammenarbeit und Entwicklung (Organisation for Economic Cooperation and Development: OECD) sollen bis zu 60 Prozent des Welthandels innerhalb multinationaler Konzerne stattfinden, was den enormen Umfang dieser Manipulationsmöglichkeiten deutlich macht. ${ }^{125}$

Nach dem OECD-Musterabkommen zur Vermeidung von Doppelbesteuerung ${ }^{1226}$, das den meisten bilateralen Doppelbesteuerungsabkommen zugrunde liegt, sind

1224 o. V.: Wie Amazon sich ums Steuerzahlen drückt. Handelsblatt vom 07.12.2012, auf http:// www.handelsblatt.com/unternehmen/dienstleister/ableger-in-luxemburg-wie-amazon-sich-ums-steuerzahlen-drueckt/7492820.html (letzter Zugriff: 20.02.2017).

1225 Neighbour, John: Transfer Pricing. Keeping it at Arms Length. OECD Centre for Tax Policy and Administration, Paris 2002.

1226 OECD: Model Tax Convention on Income and on Capital, 2008, auf: http://www. oecd-ilibrary.org/taxation/model-tax-convention-on-income-and-on-capital2008_9789264060807-en (letzter Zugriff: 17.02.2017). 
Unternehmen zur Anwendung des Fremdvergleichsprinzips verpflichtet, doch gestaltet sich die praktische Überprüfung ausgesprochen schwierig, weil die Preise nicht auf dem Markt gebildet werden..$^{1227}$

Der Bereich des gerechten und effizienten Standortwettbewerbs wird verlassen, wenn ein Unterbietungswettbewerb um die Besteuerungsanteile entsteht, der nicht mehr im Zusammenhang mit den erbrachten staatlichen Gesamtleistungen steht. ${ }^{1228}$ Um der aggressiven Steuergestaltung durch multinationale Großkonzerne entgegen zu wirken, haben die OECD und die G20-Staaten im Jahr 2013 die Initiative zur internationalen Bekämpfung von Gewinnkürzungen und Gewinnverlagerungen (Base Erosion and Profit Shifting - BEPS) gestartet. Im Oktober 2015 hat die OECD die Ergebnisse des BEPS-Projektes vorgestellt und einen Aktionsplan zur Bekämpfung von Steuerbetrug und zur Vermeidung globaler Minderbesteuerung vorgelegt. Das BEPS-Maßnahmenpaket wurde im November 2015 von den G20-Regierungschefs verabschiedet. In den OECD-Papieren werden fünfzehn Handlungsfelder - sogenannte Action Points - diskutiert, die die teilnehmenden Staaten in nationales Recht umsetzen sollen. ${ }^{1229}$

\subsection{Internationale Steuerhinterziehung}

Internationale Steuerhinterziehung wird durch eine hohe Faktormobilität begünstigt. Insbesondere Geldkapital lässt sich leicht und diskret ins Ausland verlagern und dort investieren. Wenn sich ein Staat der Kooperation mit anderen Staaten z.B. durch Informationsaustausch verweigert, fließt ihm vermehrt Geldkapital zu, das nicht erfasst ist. Ein solcher Staat kombiniert eine geringe oder nicht vorhandene Besteuerung des zugeflossenen Kapitals mit einem strengen Bankgeheimnis, sodass das zugeflossene Kapital und deren Erträgnisse möglichst nicht von anderen Staaten erfasst und besteuert werden können. Solche Staaten werden meist "Steueroasen“ genannt. ${ }^{1230}$

1227 Liebert, Nicola: Steuergerechtigkeit, S. $152 \mathrm{f}$.

1228 Valta, Matthias: Das Internationale Steuerrecht, S. 178 und S. 183.

1229 Riedlinger, Raoul: Begrüßung, in: Deutsches wissenschaftliches Institut der Steuerberater e. V. (Hrsg.): Base Erosion and Profit Shifting (BEPS) - Ein Thema für den Mittelstand. DWS - Symposium 2015. DWS-Schriftenreihe 35, Berlin 2016, S. 10.

1230 Die großen Volkswirtschaften der Welt setzten sich im Jahr 1996 im Rahmen einer koordinierten Initiative der OECD das Ziel, den schädlichen Steuerwettbewerb einzuschränken. Dabei wurden sowohl Mitgliedsländer der OECD als auch Drittstaaten untersucht. Bei den Mitgliedsländern wurden die Höhe des Effektivsteuersatzes, der 
Die Existenz und die Praktiken von "Steueroasen“ werfen ein Schlaglicht auf zwei Gerechtigkeitsprobleme in der Steuerpolitik: Auf nationaler Ebene tragen sie dazu bei zu verhindern, dass das Gleichheitsprinzip umgesetzt wird, weil sie zahlreiche Möglichkeiten zur Steuerflucht bieten. Auf internationaler Ebene ermöglichen sie Gewinnverschiebungen und Kapitalflucht, was insbesondere die schwachen Entwicklungsländer trifft, da diesen Steuereinnahmen entgehen. ${ }^{1231}$

Was machen „Steueroasen“, wie sind sie entstanden? Um dieses zu ergründen, empfiehlt es sich in die Geschichte der Schweiz als Finanzplatz in den Zwanzigerjahren des vorigen Jahrhunderts einzutauchen.

Nach dem Ersten Weltkrieg war die Staatsverschuldung in den kriegführenden Ländern explodiert, was dazu führte, dass diese dazu übergingen, große Einkommen und Vermögen zu besteuern. In Deutschland wurde mit dem von Reichsfinanzminister Matthias Erzberger geschaffenen Reichseinkommen-steuergesetz die Progression enorm verschärft; der in Preußen in der Vorkriegszeit bei vier Prozent $^{1232}$ gelegene Spitzensteuersatz wurde um das Fünfzehnfache bis auf 60 Prozent angehoben, und die Steuersätze wurden nunmehr auf alle Einkommen eines Steuerpflichtigen angewendet. Hinzu kam das im Jahr 1919 eingeführte

Geltungsbereich („ring fencing“), fehlende Transparenz und mangelhafter Informationsaustausch als Hauptkriterien für die Beurteilung herangezogen (OECD: Harmful Tax Competition - An Emerging Global Issue, 1998, https://www.oecd.org/tax/transparency/44430243.pdf (letzter Zugriff: 17.02.2017). Anm. 57 ff). Bei den Drittstaaten wurden keine schädlichen Einzelbestimmungen in den entsprechenden Steuergesetzen untersucht, sondern das Gesamtbild der Steuergesetze als Maßstab herangezogen. Maßgeblich für die Einordnung als „Steueroase“ waren die nominelle Höhe des Steuersatzes, ein mangelnder Informationsaustausch sowie keine oder nur mangelhafte Transparenz. Die OECD veröffentlichte im Jahr 2000 eine Liste von insgesamt 47 „potentially harmful preferential tax regimes“ innerhalb der OECD-Mitgliedsstaaten und von 35 „Steueroasen“, denen Gegenmaßnahmen angedroht wurden, wenn sie sich nicht verpflichteten, die unfairen Praktiken innerhalb eines bestimmten Zeitraumes zu eliminieren (OECD: Towards Global Tax Co-operation - Report to the $2000 \mathrm{Mi}$ nisterial Council Meeting an Recommendations by the Committee on Fiscal Affairs, 2000 https://www.oecd.org/tax/transparency/about-the-global-forum/publications/ towards-global-tax-cooperation-progress.pdf (letzter Zugriff: 17.02.2017). Die staatlichen Initiativen - auch durch den Abschluss von bilateralen Verträgen - haben Erfolge gebracht: Seit Mai 2009 befindet sich kein Land mehr auf der List of Uncooperative Tax Havens (OECD: List of Uncooperative Tax Havens, 2009).

1231 Liebert, Nicola: Steuergerechtigkeit, S. 179.

1232 EStG Preußen 1891: $\$ 17:$ „Bei Einkommen von mehr als 100.000 Mark bis einschließlich 105. 000 Mark beträgt die Steuer 4.000 Mark und steigt bei höheren Einkommen in Stufen von 5.000 Mark um je 200 Mark." Preußisches Einkommensteuergesetz vom 24. Juni 1891 in: FinanzArchiv 8. Jahrg., H.2 (1891), S. 331-451. 
„Reichsnotopfer“, eine Steuer mit Spitzensätzen von 65 Prozent vom Vermögen sowie eine Kriegsabgabe für Vermögenszuwächse.

Während der Französischen Revolution hatte die französische Nationalversammlung 1791 das Kataster für Frankreich geschaffen, um den gesamten Immobilienbesitz zu erfassen, seinen Wert zu ermitteln und die Privilegien des Adels und des Klerus abzuschaffen. Seit Mitte des 19. Jahrhunderts setzte sich bewegliches Vermögen gegenüber dem Grundbesitz durch. 1920 bestand das Vermögen der Reichsten im Wesentlichen aus Wertpapieren, die nicht in einem Kataster registriert wurden und daher in Depots von Banken verwaltet werden konnten. Da zwischen Schweizer Banken und dem Ausland keinerlei Informationsaustausch bestand, konnten es die Einleger unterlassen, die erzielten Zinsen und Dividenden bei ihrer Steuererklärung anzugeben. Das war die Geburtsstunde der Steuerfluchtindustrie. ${ }^{1233}$

$\mathrm{Ab}$ den 80er Jahren des vorigen Jahrhunderts war die Schweiz nicht mehr die einzige Steueroase der Welt. In Hongkong, Singapur, Jersey, Luxemburg und die Bahamas entstanden neue Zentren für Vermögensverwaltung. Waren von den Zwanziger- bis in die Siebzigerjahre des vorigen Jahrhunderts sämtliche europäischen Vermögen in die Schweiz geflossen, so erfolgte die Steuerflucht ab den Achtzigerjahren überwiegend in die neuen Offshore-Zentren in Europa, Asien und der Karibik. ${ }^{1234}$

Als weiterer Faktor zur Verschleierung von Vermögenswerten kam hinzu, dass die Kunden nicht mehr selbst Aktien und Anleihen zum Kauf aussuchten, sondern diese Aufgabe an Manager von Investmentfonds übertrugen, die das Geld in Fonds in aller Welt anlegten. Gegenwärtig haben die meisten Fonds, in die Europäer gegenwärtig investieren, ihren Sitz in den Ländern Luxemburg, Irland und auf den Kaimaninseln. Das Großherzogtum Luxemburg ist seither das Land, das nach den Vereinigten Staaten die meisten dieser Fonds beherbergt. Zucman führt ein Beispiel an, wie völlig legal verschiedene Steuern umgangen werden können: „Nehmen wir als Beispiel einen luxemburgischen Fonds, der in amerikanische Aktien investiert. Aufgrund des Steuerabkommens zwischen diesen beiden Ländern erheben die Vereinigten Staaten keine Steuern auf die ausgeschütteten Dividenden. Das Großherzogtum besteuert weder die Dividenden, die der Fonds erhält, noch die Erträge, die er an die Anleger auszahlt. Die gleiche Situation herrscht in Irland und auf den Kaimaninseln. ${ }^{{ }^{\prime 1235}}$

1233 Zucman, Gabriel: Steueroasen. Wo der Wohlstand der Nationen versteckt wird, Berlin 2014, S. $12 \mathrm{ff}$.

1234 Ebd., S. 36 f.

1235 Ebd.,: S. 39 f. 
Das Beispiel zeigt deutlich, dass es nicht nur die unterschiedlichen Steuersätze sind, sondern auch die ausgeübten Methoden der Regulierung und der Geheimhaltung gegenüber anderen Staaten, die die fiskalische Souveränität aller anderen Staaten untergraben. Die Folgen der Steuervermeidung und Steuerhinterziehung trifft zudem die Entwicklungsländer erheblich schwerwiegender als die reichen Länder, da diesen die Einnahmen für die Erfüllung der staatlichen Aufgaben fehlen, wenn Korruption im Spiel ist. ${ }^{1236}$

Wie uneffektiv die oben aufgeführten Transparenzversprechen (vgl. Fußnote 20) in der praktischen Anwendung sind, zeigte sich, als es einem internationalen Journalistenteam gelang, eine Festplatte mit einer Datensammlung aus einer anonymen Quelle zu erhalten, die bereits im Jahr 2009 US-amerikanischen, britischen und australischen Steuerbehörden angeboten worden war. Die Journalisten werteten Steuerdaten von rund 130.000 Personen aus fast allen Ländern der Welt aus und zeigten auf, wie mithilfe von Briefkastenfirmen und Offshore-Konten Steuern hinterzogen worden sind. ${ }^{1237}$ Unabhängig von diesem damals größten grenzübergreifenden Rechercheprojekt in der Geschichte des Journalismus nehmen die USA, Großbritannien, Australien und Deutschland die Auswertung der Daten in einer international koordinierten Aktion gemeinsam vor. Nach Angaben der Behörden handelt es sich unter anderem um Daten über Briefkastenfirmen, Stiftungen und Trusts auf den Cook-, den Kaimaninseln, in Singapur und auf den Britischen Jungferninseln. ${ }^{1238}$

In den bekannt gewordenen Daten tauchen alle Großbanken und zahlreiche Einzelpersonen auf, die nach einer auf Daten des Internationalen Währungsfonds (IWF) basierenden Studie vom Tax Justice Network aus dem Jahr 2012 (für das Jahr 2010) weltweit mindestens zwischen 21 Billionen und 32 Billionen Dollar Finanzvermögen in "Steueroasen“ versteckt haben sollen, um nicht besteuert zu werden. ${ }^{1239}$ Der Vorsitzende der Deutschen Steuer-Gewerkschaft e. V., Thomas Eigenthaler, schätzt, dass über 400 Milliarden Euro aus Deutschland stammendes

1236 Vgl. hierzu die Steuereinnahmen ausgewählter Länder gemessen am BIP in Liebert, Nicola: Steuergerechtigkeit, S. 208-220.

1237 Uken, Marlies: Steueroasen unterlaufen Transparenzversprechen, auf: ZEIT ONLINE vom 04.04.2013, http://www.zeit.de/wirtschaft/2013-04/steueroase-oecd-praxis-liste (letzter Zugriff: 15.02.2017).

1238 o. V.: Kampf gegen Steueroasen. Deutsche Fahnder bekommen Offshore-Daten. Spiegel Online vom 10. Mai 2013, auf: http://www.spiegel.de/wirtschaft/soziales/kampf-gegen-steueroasen-deutsche-fahnder-bekommen-offshore-daten-a-899094.html (letzter Zugriff: 20.02.2017).

1239 Henry, James S.: Neue Erkenntnisse zum Preis des Offshore-Systems. Neue Schätzungen für nicht gemeldetes Privatvermögen und Einkommen sowie für Ungleichheit und 
Schwarzgeld im Ausland angelegt ist, was nicht verwundert, denn Deutschland wird unter den zehn bedeutendsten Ländern für Steuerflucht vom Tax Justice Network aufgeführt. ${ }^{1240}$

Im November 2014 gelangten im Rahmen der „Luxemburg Leaks“ 28.000 Seiten bisher geheimer Steuerdokumente an die Öffentlichkeit, die belegen, wie internationale Unternehmen ihre Steuerflucht über Luxemburg organisieren. ${ }^{1241}$ EU-Kommissionspräsident Juncker weist die Vorwürfe zurück, in seiner Zeit als Regierungschef von Luxemburg sein Land mit Steuertricks reich gemacht zu haben.

Wir haben keine Politik gemacht in Luxemburg mit dem Ziel, anderen Ländern Steuereinnahmen wegzunehmen. Wir haben um moderne Unternehmen wie AOL, Amazon geworben. Die dazugehörigen Steuerbeschlüsse haben die Steuerbehörden getroffen, nicht die Regierung. Aber natürlich haben wir geworben und verhandelt wie andere Regierungen auch, die irische, die niederländische und auch die belgische. ${ }^{1242}$

Luxemburg, das 1957 die Europäische Wirtschaftsgemeinschaft mitbegründet hat, ist es gelungen, einer der wichtigsten Finanzplätze der Welt zu werden. Der Staat ging ab den Siebzigerjahren völlig neue Wege, er verkaufte multinationalen Konzernen der ganzen Welt das Recht, selbst über ihre Steuersätze und rechtlichen Verpflichtungen zu bestimmen. Damit lockte er Tausende Investmentfonds, multinationale Holdings, Briefkastenfirmen und Privatbanken an. ${ }^{1243}$

Unter Federführung des Netzwerks Steuergerechtigkeit forderten verschiedene Organisationen Bundesfinanzminister Schäuble in einem offenen Brief am 3. Dezember 2014 auf, alles für die Einführung eines Registers zu tun, das die Nutznießer und Eigentümer von Firmen, Stiftungen, Trusts und anderen Rechtspersonen offenlegt. Die Unterzeichner führen aus:

Mit dem Register hätten Ermittlungsbehörden, Öffentlichkeit, Medien und Wirtschaft in der EU und weltweit einen besseren Überblick - auch aus Entwicklungsländern, von deren Eliten einige die Intransparenz in der EU ausnutzen. Missstände und

entgangene Steuereinnahmen weltweit, Juli 2012, auf: www.taxjustice.net/cms/upload/ pdf/Deutsch/TJN2012 (letzter Zugriff: 20.02.2017), S.5.

1240 Meinzer, Markus: Deutschland im Schattenindex 2011, in: Informationsbrief 06 des Netzwerks Steuergerechtigkeit Deutschland vom 6. April 2012.

1241 ICIJ: Explore the Documents. Luxembourg Leaks Database, aus: https://www.icij.org/ project/luxembourg-leaks. (letzter Zugriff: 17.02.2017).

1242 Gammelin, Cerstin: Juncker widerspricht den Vorwürfen zu Steuertricks, 27.11.2014, auf: http://www.sueddeutsche.de/wirtschaft/eu-kommissionschef-jean-claude-juncker-ich-beantworte-keine-widerlichen-fragen-1.2242453-2 (letzter Zugriff: 19.02.2017).

1243 Zucman, Gabriel: Steueroasen, S. 97 f. 
Straftaten würden dadurch unterbleiben oder könnten schneller aufgeklärt werden. [...] Außerdem braucht es die Veröffentlichung aller Verstöße der Banken und anderer durch die Richtlinie verpflichteten Unternehmen. Hierfür haben sich das Europäische Parlament und die EU-Kommission ausgesprochen [...]. Nur die konsequente Offenlegung kann die Banken abschrecken. [...] Deshalb fordern wir Sie auf, sich im Rat für eine Veröffentlichung einzusetzen. ${ }^{1244}$

Die Geschichte des größten Daten-Leaks aller Zeiten wurde unter der Bezeichnung „Panama Papers“ im Frühjahr 2016 der Öffentlichkeit zugänglich gemacht. Zwei Reporter der Süddeutschen Zeitung berichteten davon, dass ihnen die Daten Hunderttausender Briefkastenfirmen anonym zugespielt worden sind, und schalteten zur Auswertung dieser Daten ein weltweites Netzwerk - das International Consortium for Investigative Journalists (ICIJ) - in Washington D. C. - ein. Gemeinsam mit dem ICIJ koordinierten sie die Arbeit von 400 Reportern aus 80 Ländern und arbeiten fast ein Jahr mit den wichtigsten Medien der Welt zusammen. ${ }^{1245}$

Die Daten der Reporter „zeigen ansatzweise, wie verstrickt fast alle wichtigen deutschen Banken in das Offshore-System waren oder sind. ${ }^{“ 1246}$ Mehr als fünfzehn deutsche Banken haben - über ihre ausländischen Tochterfirmen - offenbar aktiv und systematisch bei der Steuerhinterziehung ihrer Kunden geholfen. Bemerkenswert ist, dass die Commerzbank, die im Jahr 2008 mit 18 Milliarden Euro Steuergeldern unterstützt wurde, sich nach einer Razzia im eigenen Haus im Februar 2015 mit der Staatsanwaltschaft auf die Einstellung des eingeleiteten Strafverfahrens geeinigt hat; im Gegenzug musste die Bank 17 Millionen Euro bezahlen. Auch die HypoVereinsbank und die HSH Nordbank haben sich mit der Staatsanwaltschaft geeinigt und ebenfalls 10 bzw. 22 Millionen Euro bezahlt.. ${ }^{247}$

Es stellt sich die Frage, wie Steuerhinterziehung erfolgreich bekämpft werden kann. Der französische Wirtschaftswissenschaftler Gabriel Zucman zeigt in seinem Buch „Steueroasen - Wo der Wohlstand der Nationen versteckt wird“ auf, wie durch automatischen Informationsaustausch und durch die Schaffung eines weltweiten Wertpapierregisters die Erträge bzw. Vermögen im 21. Jahrhundert - notfalls

1244 Bsirske, Frank u. a.: Offener Brief an Dr. Wolfgang Schäuble vom 03.12.2014: EU-Anti-Geldwäsche-Richtlinie-Transparenz bei Eigentümern und Sanktionen, https://www. transparency.de/fileadmin/pdfs/Themen/Finanzmarkt/Brief_Schaeuble_AMLD_Dezember_2014.pdf (letzter Zugriff 17.02.2017).

1245 Aus den Unterlagen ergeben sich eine unheimliche Dichte von Mitgliedern der reichsten deutschen Familien. Vgl. Obermayer, Bastian/Obermaier, Frederik: Panama Papers. Die Geschichte einer weltweiten Enthüllung, Köln, 2016, S. 205.

1246 Ebd., S. 52.

1247 Ebd., S. 53 und 258. 
durch Zwang und Sanktionen - besteuert werden können. ${ }^{1248}$ Zucman verweist darauf, dass in Schweden ein solches Register bereits besteht und in Frankreich die französischen Banken seit Jahrzehnten dem Fiskus sämtliche Informationen liefern, die sie zum Einkommen ihrer Kunden haben. Die „Steueroasen“ werden jedoch nur unter Androhung von Sanktionen nachgeben; denn „kein Land kann sich gegen den gemeinschaftlichen Willen der Vereinigten Staaten und der großen Länder der Europäischen Union stellen. ${ }^{\text {1249 }}$

Zucman schlägt daher vor, ein weltweites Finanzkataster zu schaffen, das zeigt, wem die zirkulierenden Aktien, Anleihen und Fondsanteile auf der ganzen Welt gehören. Ein solches Kataster böte den Finanzverwaltungen die Möglichkeit, zu überprüfen, ob insbesondere die Offshore-Banken ihnen alle Daten übermitteln, die ihnen zur Verfügung stehen; denn nur mit einem weltweiten Finanzkataster sei eine Kontrolle der "Steueroasen“ möglich. ${ }^{1250} \mathrm{Zucman}$ schlägt weiterhin vor, eine weltweite Kapitalsteuer einzuführen, verweist aber darauf, dass ein nicht zu vernachlässigender Teil der Wertpapiere beim Finanzkataster als Besitz von Trusts ohne eindeutig benannte Eigentümer geführt werden. Er hält es sogar für denkbar, dass ein Handel großen Stils mit Strohmännern entstünde, die sich als Eigentümer der Vermögen von Steuerhinterziehern oder Drogenhändlern ausgeben könnten.

\subsection{Ausblick}

Mit der Globalisierung und der damit verbundenen Zunahme internationaler Verflechtungen gewinnt die Frage der Steuergerechtigkeit eine ganz neue Dimension. Die Privilegierung von Produktionsfaktoren im internationalen Staatenwettbewerb, die Steuergestaltung von international operierenden Unternehmen und die Kapitalflucht in Steueroasen erfordern gezielte Maßnahmen auf nationaler und internationaler Ebene, um dem Gleichheitsgrundsatz in der Staatengemeinschaft Rechnung zu tragen. Dabei kommt es im Staatenwettbewerb darauf an, dass nicht nur widerspruchsfreie Normen geschaffen werden, sondern diese auch gleichmäßig vollzogen werden.

Anthony B. Atkinson hält es für durchaus vorstellbar, dass die derzeit praktizierten Maßnahmen gegen schädliche internationale Steuerpraktiken unter dem Schutz der OECD zur Gründung einer Weltsteuerbehörde führen könnten. Atkinson

1248 Zucman, Gabriel: Steueroasen, S. $12 \mathrm{ff}$.

1249 Ebd., S. 13.

1250 Ebd., S. $102 \mathrm{ff}$. 
schlägt daher vor, den Status eines globalen Steuerzahlers zu schaffen, der Privatpersonen erlauben sollte, sich für eine globale Steuerregelung zu entscheiden. ${ }^{1251}$

Da Unternehmen von der Infrastruktur der Länder profitieren, in denen sie ihre Waren verkaufen, sollte eine Minimalsteuer für Unternehmen eingeführt werden. Diese könnte anhand von Betriebsergebnissen vor Zinsen, Steuern und Abschreibungen (EBITDA) bestimmt werden oder könnte sich auch am Wert der Umsatzerlöse im Steuerhoheitsgebiet orientieren. ${ }^{1252}$

Die BEPS-Initiative der OECD ist in erster Linie ein Vorkommnis großer, nicht inhabergeführter Unternehmen. Die Bekämpfung von BEPS dient somit der Wiederherstellung einer Größenneutralität und ist eher als hilfreich als schädlich anzusehen. Die große Stärke am BEPS-Projekt ist, dass es ein multilateral initiiertes und abgestimmtes Programm ist, das zu einer gravierenden Änderung des internationalen Steuerrechts führen wird. ${ }^{1253}$

Die Initiativen von internationalen Journalisten zeigen auf, dass es möglich ist, an steuerlich relevante Informationen weltweit zu gelangen und diese zu veröffentlichen, sodass eine Verfolgung von Steuerhinterziehern im großen Stil möglich wurde. Um „Steueroasen“ zu besiegen, schlägt Zucman vor, Steuerfragen ins Zentrum der Handelspolitik zu rücken, denn „mit dem Finanzkataster und der Quellensteuer auf Kapital könnten die Staaten einen Teil der Souveränität wiedererlangen, die sie sich haben nehmen lassen, um die Explosion der Steuerungerechtigkeiten einzudämmen. ${ }^{\text {"1254 }}$

1251 Atkinson, Anthony B.: Ungleichheit. Was wir dagegen tun können, Stuttgart 2016, S. 260 .

1252 Ebd., S. $262 \mathrm{f}$.

1253 Vgl. im Einzelnen: Rautenstrauch, Gabriele/Prinz zu Hohenlohe, Franz: KPMG International: Der BEPS Aktionsplan vom 02.03.2016, auf: https://home.kpmg.com/de/ home/themen/2016/03 /beps-aktionsplan. ᄀhtml (letzter Zugriff: 17.02.2017); Reimer, Ekkehart/Heyes, Martin: Base Erosion and Profit Shifting (BEPS) - Ein Thema für den Mittelstand, in: Deutsches wissenschaftliches Institut der Steuerberater e. V. (Hrsg.): Base Erosion and Profit Shifting (BEPS) - Ein Thema für den Mittelstand, DWS - Symposium 2015. DWS-Schriftenreihe 35, Berlin 2016, S. 17-65, S. 43 f.

1254 Zucman, Gabriel: Steueroasen, S. 14 und 110. 
Dieses Buch wird unter der Creative Commons Namensnennung 4.0 International Lizenz (http://creativecommons.org/licenses/by/4.0/deed.de) veröffentlicht, welche die Nutzung, Vervielfältigung, Bearbeitung, Verbreitung und Wiedergabe in jeglichem Medium und Format erlaubt, sofern Sie den/die ursprünglichen Autor(en) und die Quelle ordnungsgemäß nennen, einen Link zur Creative Commons Lizenz beifügen und angeben, ob Änderungen vorgenommen wurden.

Die in diesem Buch enthaltenen Bilder und sonstiges Drittmaterial unterliegen ebenfalls der genannten Creative Commons Lizenz, sofern sich aus der Abbildungslegende nichts anderes ergibt. Sofern das betreffende Material nicht unter der genannten Creative Commons Lizenz steht und die betreffende Handlung nicht nach gesetzlichen Vorschriften erlaubt ist, ist für die oben aufgeführten Weiterverwendungen des Materials die Einwilligung des jeweiligen Rechteinhabers einzuholen.

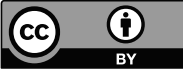

